\title{
Associer chercheurs et praticiens à la conception d'outils didactiques ou de dispositifs innovants pour améliorer l'enseignement
}

\section{Roland Goigoux}

\section{(2) OpenEdition}

\section{Journals}

Édition électronique

URL : http://journals.openedition.org/educationdidactique/2872

DOI : 10.4000/educationdidactique.2872

ISSN : 2111-4838

Éditeur

Presses universitaires de Rennes

\section{Édition imprimée}

Date de publication : 31 décembre 2017

Pagination : 135-142

ISBN : 978-2-7535-7319-2

ISSN : 1956-3485

\section{Référence électronique}

Roland Goigoux, «Associer chercheurs et praticiens à la conception d'outils didactiques ou de dispositifs innovants pour améliorer l'enseignement », Éducation et didactique [En ligne], 11-3 | 2017, mis en ligne le 31 décembre 2017, consulté le 14 septembre 2020. URL : http://

journals.openedition.org/educationdidactique/2872 


\section{ASSOCIER CHERCHEURS ET PRATICIENS À LA CONCEPTION D'OUTILS DIDACTIQUES OU DE DISPOSITIFS INNOVANTS POUR AMÉLIORER L'ENSEIGNEMENT}




\section{INTRODUCTION}

Dans l'article soumis au débat, Anthony Bryk résume les principales propositions de la fondation Carnegie sur la conduite des réformes visant l'amélioration de l'enseignement (Bryk, Gomez, Grunow \& LeMathieu, 2015). Ces propositions, mêmes si elles sont singulières dans leur expression, reposent sur des principes partagés par grand nombre de chercheurs en éducation aux États-Unis. Elles visent à produire des améliorations mesurables, ce qui les distingue de celles des sciences de l'éducation françaises qui répugnent encore trop souvent à évaluer l'impact des pratiques qu'elles décrivent et des innovations qu'elles promeuvent. Les réformes souhaitées par la fondation Carnegie reposent sur des dispositifs innovants élaborés pour répondre aux préoccupations professionnelles des enseignants en tenant compte des connaissances scientifiques avérées et disponibles. Les démarches préconisées s'éloignent donc, d'une part, des conceptions applicationnistes basées sur la transposition des connaissances fondamentales élaborées par les sciences cognitives et, d'autre part, des recherches-actions centrées sur des préoccupations si locales et situées qu'elles ne se donnent pas les moyens d'une généralisation ou d'une décontextualisation des connaissances produites. Les propositions résumées dans la figure 5 de l'article ${ }^{1}$ indiquent le changement de paradigme qui s'est opéré progressivement notamment à la suite des rapports du conseil national de la recherche (NCR, 1999, 2004). Nous insisterons ici sur la profonde modification des relations entre décideurs, chercheurs et praticiens qu'implique ce changement, modification que nous appelons de nos vœux en France mais qui a bien du mal à émerger.

\section{DE NOUVELLES RELATIONS ENTRE DÉCIDEURS, CHERCHEURS ET PRATICIENS}

Accompagnée et théorisée par le SERP ${ }^{2}$ (The Strategic Education Research Partnership), un organisme créé par l'académie des sciences américaine, la promotion de nouveaux partenariats en éducation s'apparente à celui qui existe entre la recherche et la pratique dans les domaines de la médecine, l'agriculture ou le développement durable. Ce renouvellement est apparu nécessaire à la suite des bilans décevants des réformes et des innovations pédago- giques y compris celles qui étaient basées sur une approche Evidence-based : les promesses d'améliorations ne sont pas plus tenues aujourd'hui qu'hier. Dès 1995, Tyack et Cuban avaient analysé ces échecs et les avaient attribués à l'insuffisante prise en compte des contextes d'enseignement et des habitus professionnels des enseignants (leur Grammar of schooling que nous appelons « genre professionnel »; Clot \& Faita, 2000 ; Goigoux, 2007) dans la mise en œuvre des réformes pédagogiques. Ils avaient montré, par exemple, que les chances de réussite d'une innovation dépendaient d'une part de sa compatibilité avec les schèmes professionnels des enseignants, ce qui implique de produire des connaissances à leur sujet, et, d'autre part, de l'efficience de l'intervention, c'està-dire du rapport entre ses coûts pour les enseignants (réorganisation cognitive, surcharge de travail, investissement émotionnel...) et ses bénéfices (satisfaction due à l'amélioration des apprentissages des élèves ou à l'accroissement du sentiment de compétence du professeur). Bryk surenchérit et explique que les «bonnes idées » mises en œuvre trop rapidement et sans souci de leur intégration dans la diversité des contextes professionnels échouent faute de stratégie adossée à une théorie du changement des pratiques professionnelles (comme ce fut le cas par exemple en France avec l'innovation conduite au cours préparatoire par Gentaz et collaborateurs, 2013). Les décideurs, écrit-il, négligent souvent un facteur décisif : l'engagement des acteurs de terrain, enseignants et cadres, dans les projets innovants. Autrement dit, ils ne prennent pas suffisamment en compte les futurs utilisateurs lorsqu'ils conçoivent de nouveaux dispositifs pédagogiques.

Ces critiques ont conduit les Américains à promouvoir un nouveau type de recherche sur l'enseignement, des recherches "intégrées à la pratique » appelées PEER (Pratice Embedded Educational Research). Visant l'amélioration des apprentissages des élèves, elles reposent sur quatre principes (Donovan, Wigdor \& Snow, 2003) que les sciences de l'éducation françaises gagneraient à reprendre à leur compte comme nous le faisons nous-mêmes depuis quinze ans. 


\section{QUATRE PRINCIPES POUR DES RECHERCHES INTÉGRÉES À LA PRATIQUE}

\section{Reconnaitre l'organisation systémique de toute réforme scolaire}

Les élèves et les enseignants sont insérés dans des organisations complexes interdépendantes (pour l'école élémentaire par exemple : la classe, l'école, l'environnement scolaire, la circonscription primaire, la direction départementale de l'Éducation nationale, l'État...), ce qui implique que les améliorations visées sur les apprentissages des élèves tiennent compte des contraintes qui pèsent sur l'organisation du travail enseignant. Les décideurs et les cadres sont souvent impatients d'obtenir des résultats à court terme et n'accordent pas aux pratiques novatrices le temps dont elles ont besoin pour être maîtrisées, adaptées, évaluées et institutionnalisées. C'est ainsi que le dispositif français "Plus de maitres que de classes » a mis quatre ans pour atteindre sa maturité : une première année a été consacrée aux problèmes de réorganisation du travail collectif au sein des écoles, les deux années suivantes à des améliorations didactiques avant qu'on constate en quatrième année une accentuation de l'attention portée aux élèves en grande difficulté $e^{3}$. Il est regrettable que ce dispositif ait été remplacé par un autre dans les réseaux d'éducation prioritaire avant même d'avoir été évalué. Les décideurs français ne sont pas encore des adeptes du paradigme de l'amélioration.

\section{Construire un partenariat structuré et soutenu entre les praticiens et les chercheurs}

Pour cela, il est indispensable de considérer que chacun de deux ensembles d'acteurs est détenteur et producteur de connaissances d'égale valeur et d'égale importance même si elles sont de nature différente. Dans ce modèle de partenariat, les chercheurs doivent faire l'effort de connaître les réalités de la pratique autant que les praticiens doivent identifier les exigences de la recherche. Les difficultés inévitables (par exemple pour construire des expérimentations randomisées dans des environnements complexes) devraient être considérées comme des problèmes à partager et à résoudre ensemble (Donovan, Snow et Daro, 2013). C'est dans cet esprit que nous nous sommes engagés dans la création d'un institut Carnot de l'Éducation en région AuvergneRhône-Alpes (ICÉ-AuRA) que nous animons conjointement avec Pascal Bressoux sous la direction d'Alain Trouillet. Dans cet institut, nous œuvrons pour que les projets réalisés en collaboration offrent à chaque groupe l'occasion de mieux comprendre les attentes, les ressources et les contraintes de l'autre.

\section{Partir des préoccupations des praticiens pour déterminer les problématiques de recherche}

Cela implique de modifier la logique actuelle des communautés de recherche qui décident seules des problèmes à aborder en fonction du développement des connaissances scientifiques dans leur domaine (Snow, 2015). Bien que le projet d'institut Carnot de l'éducation ait été initié pour favoriser les démarches ascendantes et faire émerger de nouvelles questions de recherche à partir des problèmes professionnels rencontrés par les enseignants ou les autres membres de la communauté scolaire, le fonctionnement de l'institut n'est pas encore à la hauteur de cette ambition. Les schémas applicationnistes sont vivaces (Fougères, 2015) et les mécanismes de financement sont inadaptés à une recherche « intégrée à la pratique ». La négociation des questions initiales et la constitution des collectifs chercheurs / praticiens représentent un investissement en temps considérable qui doit être financé pour que l'entreprise soit viable. Les rectorats de la grande région AuvergneRhône-Alpes ont fait un premier pas en ce sens en recrutant des passeurs (enseignants expérimentés titulaires d'un doctorat) pour faciliter la constitution de ces collectifs et diffuser les résultats des recherches. Il reste à présent à lancer et à financer des appels à projets qui exigent des réponses conjointes chercheurs / praticiens préparées en amont.

\section{Étudier attentivement la façon dont les innovations sont conduites et traiter les variations de leur mise en ouvre comme une source majeure d'information}

Pour envisager la diffusion ou la généralisation d'une expérimentation, il est indispensable de savoir quels aspects d'un nouveau dispositif sont faciles ou difficiles à mettre en œuvre, lesquels sont adoptés rapidement ou lentement et progressivement par les 
enseignants, lesquels ont besoin d'un accompagnement par des conseillers pédagogiques ou peuvent se développer de manière autonome au sein de chaque école ou établissement, lesquels sont acceptés ou rejetés par les enseignants et pour quelles raisons (Snow, 2015).

Ce quatrième point mérite d'être développé car c'est peut-être celui qui pointe le plus nettement les limites des approches evidence-based. Même si certains décideurs sont tentés de les utiliser de façon normative, il est généralement admis que ces approches n'ont pas vocation à définir les politiques scolaires mais seulement à les éclairer (Nutley et al., 2003). Pour cela, les décideurs ont besoin de connaitre les sources de la variabilité des effets des réformes qui visent l'amélioration des apprentissages scolaires. Celles-ci génèrent souvent des effets différents selon les contextes sociaux et géographiques, l'organisation de l'école, le niveau initial des élèves, l'accompagnement de l'innovation et, surtout, l'implication des enseignants dans le processus d'innovation. Or, souligne Brik, les essais randomisés ne sont pas conçus pour apporter des informations sur tous ces points et, de manière générale, sur les conditions à réunir pour qu'une innovation soit efficace. Ils nous éclairent sur le fait qu'une intervention peut fonctionner mais pas sur les conditions requises pour qu'elle fonctionne de manière fiable dans différents contextes ou pour qu'elle ait des effets durables et significatifs sur les divers publics visés. Bon nombre d'innovations qui obtiennent des effets moyens significatifs échouent dans certaines écoles. L'examen des cas problématiques (les « déviants négatifs ») peut permettre d'identifier les facteurs explicatifs de ces phénomènes et les « conditions aux limites » pour les lieux d'intervention, les conditions ou les sous-groupes d'élèves pour lesquels ces innovations ne sont pas pertinentes. De la même manière, les conditions ayant favorisé des progrès supérieurs à la moyenne dans d'autres écoles (les " déviants positifs») devraient être analysées pour formuler de nouvelles hypothèses sur les sources d'amélioration (Pascale, Sternin \& Sternin, 2010). L'objectif final est de réduire la part des effets nuls ou négatifs d'une innovation pour en optimiser l'impact. En résumé, traiter la variation des effets comme une source de production de connaissances scientifiques, nous conduit, dans nos propres recherches, à ne plus considérer les écarts aux modèles prescrits comme des erreurs à corriger mais comme un moyen de faire évoluer les dispositifs expérimentés. Pour nous, comme pour les chercheurs impliqués dans le PEER, le processus de conception doit être itératif et participatif. Nous l'appelons, à la suite des travaux d'ergonomie de langue française (Beguin \& Cerf, 2004 ; Beguin, 2005 ), « conception continuée dans l'usage ».

\section{CONCEVOIR DE NOUVEAUX OUTILS OU DISPOSITIFS INNOVANTS DANS LE CADRE D'UN NOUVEAU TYPE DE COLLABORATION CHERCHEURS-PRATICIENS}

La démarche que nous avons élaborée pour concevoir des outils didactiques capables d'aider les professeurs à améliorer leur enseignement (Goigoux \& Cèbe, 2009) n'est pas très éloignée de celles inspirée par le National Research Council (NRC, 2004) et développée par les chercheurs du mouvement Design-Based Implementation Research (2003) (Fishman, Penuel, Allen \& Cheng, 2013) (Voir Class $\&$ Schneider, 2013 pour une présentation en français de la « Recherche Design en éducation »). Notre démarche peut être résumée en trois étapes dans lesquelles les rôles respectifs des chercheurs et des enseignants évoluent.

\section{Première étape : concevoir un prototype}

Même si ce sont les problèmes professionnels des enseignants qui sont à l'origine du projet de conception de nouveaux outils, ce sont les chercheurs spécialisés dans le domaine qui initient le processus en prenant appui sur trois principales sources de connaissances. Ils font tout d'abord une synthèse de celles qui ont trait aux processus d'apprentissage et aux difficultés des élèves dans le domaine concerné, ce qui leur permet de d'affiner les cibles de l'outil (les compétences à enseigner). Ils dressent ensuite un état des pratiques ordinaires des enseignants et ils analysent les indices qui permettent d'identifier leur zone proximale de développement professionnel. Ils élaborent ainsi un premier modèle théorique de l'utilisateur (Rabardel \& Pastré, 2005) qui détermine les contours de l'outil. Ils dressent enfin un tableau des techniques qui ont déjà fait la preuve de leur efficacité dans le domaine considéré, ce qui facilite le choix de celles qui seront retenues. Ce prototype représente 
donc un premier compromis entre ce qui pourrait apparaitre souhaitable du point de vue des apprentissages des élèves et ce qui semble raisonnable du point de vue de l'action pédagogique contextualisée. Bref, il tente de respecter les trois critères fondamentaux de l'ergonomie de conception : l'utilité, l'utilisabilité et l'acceptabilité d'un nouvel artefact par ses futurs utilisateurs.

\section{Deuxième étape : la co-conception}

Au terme de cette première étape, un prototype est soumis à un groupe de praticiens qui vont devenir co-concepteurs. Un exemple, celui du dernier chantier de ce type conduit avec Sylvie Cèbe (Cèbe $\&$ Goigoux, 2017), va nous permettre d'expliquer le déroulement de cette étape. En 2014-2015, nous avons proposé à un groupe de dix enseignantes volontaires un prototype de scénario didactique ayant pour but de développer des compétences narratives chez de très jeunes élèves. Elles l'ont essayé dans leurs classes et ont noté, à la fin de chaque séance, le temps qu'elles y avaient consacré, leurs étonnements et leurs réussites, les difficultés qu'elles avaient rencontrées, les changements, les suppressions et les ajouts qu'elles avaient réalisés. Ces premiers essais ont permis d'ouvrir le dialogue entre enseignantes et chercheurs, puis, très vite, de distinguer les propositions didactiques immédiatement adoptées par les enseignantes de celles qui ne l'ont été qu'après une longue et difficile appropriation. La discussion a porté sur les manières de faciliter et d'accélérer la compréhension et sur la maniabilité de l'outil. Elle a aussi permis d'identifier les propositions qui ont été unanimement rejetées et qu'il convenait de modifier ou de supprimer. Ce premier recueil de données nous a fait revoir la programmation de nos séances et leur durée, imaginer de nouvelles tâches et activités, en supprimer d'autres et de ritualiser plusieurs déroulements de tâches. Nous avons alors rédigé un deuxième prototype qui a été testé par vingt nouveaux enseignants avec lesquels nous avons procédé de la même manière : des observations en classe et des rencontres régulières (2015-2016). Nous avons fait de nouveaux compromis entre nos projets initiaux et les contraintes identifiées par les utilisateurs pour rédiger un troisième prototype qui a été proposé à deux cents autres enseignants (2016$2017)^{4}$. Ces derniers nous ont, eux aussi, fait part de leurs observations et nous ont conduits à procéder à d'ultimes modifications.

En résumé, la deuxième étape de la démarche repose sur un dialogue entre initiateurs du projet et utilisateurs, dialogue qui constitue le moteur de la conception. L'enjeu est d'explorer, dans un même cadre, les logiques et les positions hétérogènes des enseignants et des concepteurs pour faire œuvre commune. C'est en cela que nous parlons de co-concepteurs car les enseignants impliqués dans cette démarche jouent un rôle actif qui réduit progressivement l'asymétrie initiale. Ce modèle de la conception mobilise des savoirs hétérogènes mais qui sont jugés également légitimes. En cas de désaccord, les chercheurs tentent de ne pas user de leur autorité. Ils s'efforcent de faire de ces désaccords le vecteur de la modification de l'outil en cours de conception : on change les critères, on ajuste les spécifications, on redéfinit les finalités pour que la solution soit acceptable au sein du groupe des co-concepteurs. Dans cette démarche, c'est à la complexité du réel qu'on attribue la difficulté des échanges, pas à l'hétérogénéité des savoirs ou des points de vue (Beguin, 2013).

\section{Troisième étape : l'évaluation}

Une fois terminé, l'outil confectionné est testé en comparant les progrès des élèves de groupes expérimentaux et de groupes témoins. Les exigences méthodologiques à respecter sont différentes selon le niveau de preuve que l'on souhaite atteindre. Les arguments n'auront pas le même poids s'ils sont fondés sur : i) des suivis de cohortes ou des études de cas dans un dispositif contrôlé, ii) des expérimentations dans des situations représentatives permettant des tests d'hypothèses avec validation statistique, iii) des essais randomisés contrôlés (expérimentation avec assignation aléatoire). Dans l'exemple ci-dessus, une expérimentation de très grande ampleur (nombre de classes et durée de l'expérience) a permis de bâtir des échantillons appareillés et de contrôler plusieurs des sources de variation des effets : la durée consacrée à l'enseignement dans les trois groupes expérimentaux (un biais classique est l'accroissement du temps d'enseignement dans les groupes expérimentaux par comparaison aux pratiques ordinaires), la fidélité dans la mise en œuvre des scénarios proposés, l'âge des enfants, l'expérience des enseignants et l'intensité de leur travail collectif, le cumul des 
expériences d'enseignement (étude longitudinale sur deux ans avec usage de six scénarios d'un mois chacun) et le rôle de l'accompagnement des enseignants novateurs par les équipes de formateurs de leurs circonscriptions, etc.

En conclusion, nous nous réjouissons des convergences qui se dessinent au plan international dans le domaine des recherches sur l'amélioration de l'enseignement. L'utilisation de nouveaux outils ou dispositifs apparait de plus en plus nettement comme un vecteur d'évolution du pouvoir d'agir des professeurs. Qu'il s'agisse du choix des tâches, de leur ordonnancement dans le temps ou de la mise en œuvre des moyens de leur réalisation, la qualité de l'enseignement est largement tributaire des instruments dont disposent les maitres. Leur évolution peut donc influencer l'activité des professeurs et modifier la manière dont ils guident les apprentissages de leurs élèves. C'est pourquoi il nous semble nécessaire de poursuivre le travail de transposition des savoirs issus de la recherche en savoirs pour l'action. Les chercheurs ne peuvent pas déléguer entièrement cette tâche aux enseignants, ils doivent la réaliser avec eux.

\section{NOTES}

1. Schéma « Le paradigme de l'amélioration » diffusé en français par le centre Alain Savary au mois de juin 2016 : [http://centre-alain-savary.ens-lyon. fr/CAS/nouvelles-professionnalites/enseignants/ de-12019evidence-based-practise-aux-practice-basedevidences-et-si-les-resultats-anglo-saxons-nourrissaientdes-debats-educatifs-feconds-en-france

2. [http://serpinstitute.org/].

3. Cf. le rapport du comité de suivi du dispositif [http:// cache.media.education.gouv.fr/file/2017/09/3/Note-duComite-national-de-suivi-du-dispositif-Plus-de-maitresque-de-classes_694093.pdf].

4. Dans le cadre d'une recherche financée par l'Institut Carnot de l'Éducation, le rectorat de l'académie de Clermont-Ferrand, la direction générale de l'enseignement scolaire au ministère de l'Éducation nationale (DGESCO) et avec le soutien du centre Alain Savary - Institut français de l'Éducation (ENS Lyon).

\section{RÉFÉRENCES}

Béguin, P., \& Cerf, M. (2004). Formes et enjeux de l'analyse de l'activité pour la conception des systèmes de travail. @ctivité, 1(1). Repéré à : [http://www.activites. org/vlnl/beguin.pdf].

Béguin, P. (2005). Concevoir pour les genèses professionnelles. Dans P. Rabardel \& P. Pastré (Éds.), Modèles du sujet pour la conception. Dialectiques, activités, développement (p. 32-52). Toulouse : Octarès.

Béguin, P. (2013). La conception des instruments comme processus dialogique d'apprentissages mutuels. Dans P. Falzon (Ed.), Ergonomie constructive (p. 147-160). Presses universitaires de France, DOI [10.3917/puf. falzo.2013.01.0147].

Cèbe, S., \& Goigoux, R. (2017). Narramus. Apprendre à comprendre et à raconter. Paris : Retz

Class, B., \& Schneider, D. (2013). La Recherche Design en éducation : vers une nouvelle approche? Frantice. net, 7, 5-16.

Clot, Y., \& Faita, D. (2000). Genres et styles en analyse du travail : concepts et méthodes. Travailler, 4(7), 43.

Design-Based-Research-Collective (2003). Design-based research: An emerging paradigm for educational inquiry. Educational researcher, 32(1), 5-8.

Donovan, M. S., Snow, C., \& Daro, P. (2013). The SERP approach to problem-solving research, development, and implementation. Dans B. Fishman, W. R. Penuel, A. Allen \& B. Cheng (Eds.), Designbased implementation research: Theories, methods, and exemplars (p. 400-425). Chicago, IL : National Society of the Study of Education.

Donovan, M. S., Wigdor, A. K., \& Snow, C. E. (Eds.) (2003). Strategic education research partnership. Commmittee on a strategic education research partnership. Washington, DC : The National Academies Press.

Fishman, B. J., Penuel, W. R., Allen, A. R., Cheng, B. H., \& Sabelli, N. O. R. A. (2013). Design-based implementation research: An emerging model for transforming the relationship of research and practice. National Society for the Study of Education, 112(2), 136-156.

Fougères, R. (2015). Rapport final de la mission Institut Carnot de l'éducation (Définition, expérimentation, essaimage), remis à Madame la ministre de l'Éducation nationale de l'Enseignement supérieur et de la Recherche. Repéré à : [http://cache.media.education.gouv.fr/file/2016/51/6/rapport-fougeres-ice-juillet-2016_751516.pdf].

Gawande, A. (2010). Checklist Manifesto, The (HB). Penguin Books India.

Gentaz, E., Sprenger-Charolles, L., Colé, P., Theurel, A., $\&$ Gurgan, M. (2013). Évaluation quantitative d'un entrainement à la lecture à grande échelle pour des enfants de CP scolarisés en réseaux d'éducation prioritaire: apports et limites. Approche Neuropsychologique des Apprentissages chez l'Enfant (ANAE), 123, 172-181.

Goigoux, R. (2007). Un modèle d'analyse de l'activité des enseignants. Éducation E Didactique, 1(3), 47-69. 
Goigoux, R., \& Cèbe, S. (2009). Un autre rapport entre recherche, pratique et formation. Les instruments didactiques comme vecteur de transformation des pratiques des enseignants. Réseau Éducation et Formation. Repéré à : [https://halshs.archives-ouvertes.fr/halshs-00936348].

National Research Council (1999). How People Learn: Bridging Research and Practice. Washington, DC : The National Academies Press. Repéré à : [https://doi. org/10.17226/9457].

National Research Council (2004). Learning and instruction: A SERP research agenda. National Academies Press.

Nutley, S., Walter, I., \& Davies, H. T. (2003). From knowing to doing: a framework for understanding the evidence-into-practice agenda. Evaluation, 9(2), 125-148.

Pascale, R., Sternin, J., \& Sternin, M. (2010). The power of positive deviance. Harvard Business School Publishing, Boston, MA.

Rabardel, P., \& Pastré, P. (Eds) (2005). Modèles du sujet pour la conception. Toulouse : Octares Éditions.

Snow, C. E. (2015). Rigor and Realism: Doing Educational Science in the Real World. Educational Researcher, 44(9), 460-466.

Tyack, D. B., \& Cuban, L. (1995). Tinkering toward utopia. Harvard University Press. 